\title{
FUNÇÃO VÍSUO-ESPACIAL EM PARALISIA CEREBRAL E PROPOSTA DE NOVO TESTE COM A AJUDA DE COMPUTADOR
}

\author{
MARILISA M. GUERREIRO * - M. VALERIANA L. DE MOURA-RIBEIRO **
}

\begin{abstract}
RESUMO - Os autores apresentam o estudo da função vísuo-espacial em uma criança com paralisia cerebral (PC). Apoiados nas teorias de Luria e Piaget, justificam a existência de tal distúrbio em PC. A criança-propósito deste estudo apresentou elementos teórico-práticos que embasaram esta idéia. Face às limitações dos testes disponíveis, propomos novo teste aplicável tanto em papel quanto em computador. Trata-se de quatro linhas dispostas equi- $\neg$ distantemente em diferentes inclinações. A simplicidade do teste permitiu seu uso em computador, o que ampliou e aprofundou a investigação, acrescentando novos elementos ao estudo.
\end{abstract}

RESUMO - Os autores apresentam o estudo da função vísuo-espacial em uma criança com paralisia cerebral (PC). Apoiados nas teorias de Luria e Piaget, justificam a existência de tal distúrbio em PC. A criança-propósito deste estudo apresentou elementos teórico-práticoc. que embasaram esta idéia. Face às limitações dos testes disponíveis, propomos novo teste aplicável tanto em papel quanto em computador. Trata-se de quatro linhas dispostas equidistantemente em diferentes inclinações. A simplicidade do teste permitiu seu uso em computador, o que ampliou e aprofundou a investigação, acrescentando novos elementos ao estudo.

Visuo-spatial function in cerebral palsy and proposition of a new test with computer aid.

SUMMARY - The first idea of this study is trat there may be a disturbance of the visuo- $\neg$ -spatial function in cerebral palsy. This idea is based upon the theories of Luria and Piaget. The second idea of this study is that the computer can help the investigation of visuo-spatial function. With the intention of evaluating the spatial function in cerebral palsy, the literature related to these themes was revised. We justify the proposal of a new test to study visuo-spatial function. This test uses straight lines in four different orientations and the subject has to recognize and arrange them individually or sets of them. This was done

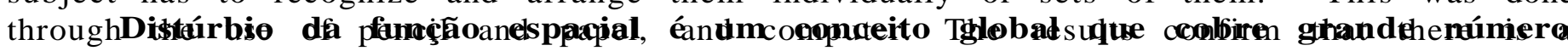

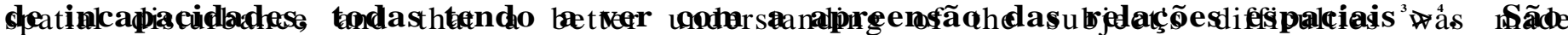

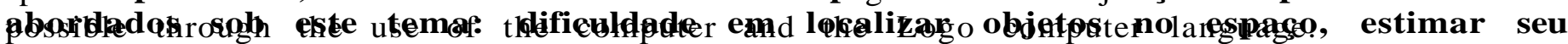
tamanho e julgar sua distância; memória prejudicada para a localização de objetos ou lugares; incapacidade para traçar um caminho ou seguir uma rota; e, dificuldade nas atividades vísuo-construtivas, por exemplo, em desenhos ${ }^{2}$. Podem ser considerados sinônimos termos como agnosia espacial, apraxia construtiva, distúrbios da percepção vísuo-espacial e cegueira espacial, entre outros. Critchley 6 revisou as características dos desenhos de pacientes com agnosia espacial e notou que há tendência a amontoar esses desenhos em um canto da página, a cópia geralmente é menor que o modelo, há linhas trêmulas e onduladas, eventualmente há reversão em espelho, há tendência a desenhar linhas verticais obliquamente e a persistir em uma única dimensão. Abercrombie ${ }^{1}$ trabalhou com crianças com paralisia cerebral (PC) e referiu que algumas dessas crianças apresentam distúrbio da percepção espacial. A PC é conceituada como o conjunto de distúrbios do movimento e da postura decorrentes de defeito ou lesão em cérebro imaturo 5,12,14.

O objetivo deste estudo é avaliar a função vísuo-espacial em uma criança com PC, pois a análise preliminar do caso sugeria haver distúrbio dessa função. Aber-

Disciplina de Neurologia Infantil, Departamento de Neurologia, Faculdade de Ciências Médicas, Universidade Estadual de Campinas (UNICAMP): * Professor Assistente; ** Professor Associado.

Dra. Marilisa M. Guerreiro - Departamento de Neurologia, Faculdade de Ciências Médicas, UNICAMP - Caixa Postal 6111 - 13081 Campinas SP - Brasil. 
crombie ${ }^{1}$ salientou a escassez existente de testes padronizados para crianças normais com os quais se possa comparar a performance do paralítico cerebral. Revisando a literatura sobre avaliação da função vísuo-espacial, verificamos o fato de que os testes disponíveis foram criados para serem aplicados em adultos. Alguns apresentam adaptações para a infância, entretanto não há padronização por idade cronológica e tampouco há padronização por idade mental. Face a essas limitações e da motivação em se atingir o objetivo, resultou a proposta do novo teste descrito em seguida, aplicável tanto em papel quanto em computador.

\section{MATERIAL E MÉTODOS}

(A) Instrumentos - Utilizaram-se, para os procedimentos com papel, folhas de papel sulfite brancas, lápis preto e cartolina branca recortada em cartões do tamanho da folha sulfite: Cartão A contendo um traço vertical de $4,5 \mathrm{~cm}$ no centro; Cartão B ,um traço horizontal de $4,5 \mathrm{~cm}$ no centro; Cartão C, um traço oblíquo para a direita de 4,5 cm no centro; Cartão D, um traço oblíquo para a esquerda de $4,5 \mathrm{~cm}$ no centro; Cartão E, quatro traços de $4,5 \mathrm{~cm}$ dispostos equidistantemente um do outro (Fig. IA). Para os procedimentos junto ao computador, utilizaram-se dois computadores da linha MSX.

(JB) Metodologia - I. Para o reconhecimento da percepção espacial foi colocado à frente da criança o cartão $\mathrm{E}$ e apresentado o cartão A, solicitando-se que apontasse no cartão E o traço igual ao do cartão A; o mesmo procedimento foi utilizado na apresentação sucessiva dos cartões B, C e D - II. Colocou-se uma folha sulfite limpa à frente da criança juntamente com um lápis e apresentou-se ocartão A; solicitou-se que reproduzisse o traço no papel à sua frente, preservando o tamanho e o local do modelo; imediatamente após a execução da tarefa proposta, trocou-se a folha sulfite por outra limpa e apresentou-se o cartão B; o mesmo procedimento foi utilizado na apresentação sucessiva dos cartões $C$, $D$ e E III. No procedimento de execução dos testes planejados junto ao computador, utilizou-se a linguagem Logo, na qual a pessoa se comunica com a máquina por comandos que movimentam uma «tartaruga» representada na tela do computador por pequeno desenho de tartaruga; utilizando apenas quatro comandos - pf (parafrente), pt (piaratrás), pd (paradireita) e pe (paraesquerda) - pode-se desenvolver uma série de atividades e desenhos na tela 17; para a execução sucessiva de cada traço projetou-se no centro da tela de um computador um traço de cada vez e foi solicitado que a criança o reproduzisse em outra tela à sua frente; a seguir, foram projetados os quatro traços ao mesmo tempo em uma das telas e, na tela à frente da criança, foi apresentada uma tartaruga no canto inferior direito, solicitando-se que reproduzisse os quatro traços, mantendo a disposição, tamanho e inclinação deles.
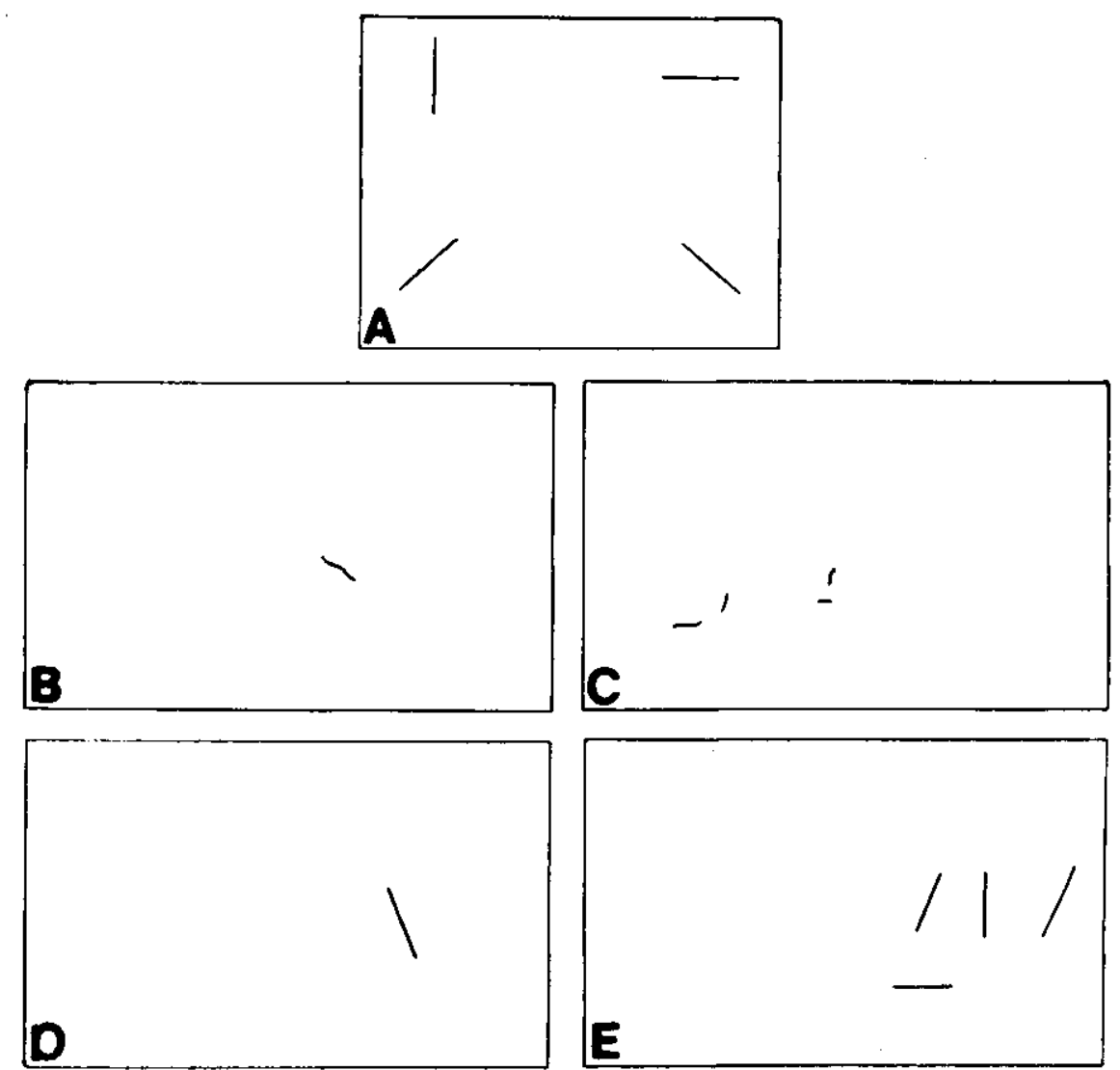

Fig. 1 - A. Cartão E: Quatro tracos de $4,5 \mathrm{~cm}$ dispostos equidis. tantemente. - B. Cópia com linho ondulada $e$ tamianho menor que o do modelo. - C. Ha amontoamento dos traços num canto do página, reversão em espelho do traco obliquo para a esquerda, além dos itens observados em $B$. - D. Ha reversão em espelho do traco individual $e$ a nāo distribuição do traço no local solicitado. - E. Ha amontoamento dos tracos num canto da tela $e$ as relacōes topográficas não foram preservadas. 


\section{OBSERVAÇÃO}

TM, trata-se de menina de 9 anos, quarta filha de casal não consanguíneo, nascida prematuramente de parto normal, hospitalar, com peso de $1900 \mathrm{~g}$. Houve choro imediato, porém há suspeita de ter havido alguma intercorrência logo após o nascimento, pois a criança encontrava-se em incubadora, com sonda nasogástrica e recebendo infusão intravenosa poucas horas após o parto. Apresentou boa evolução, recebendo alta aos 28 dias de vida com peso de $2230 \mathrm{~g}$ e sem qualquer medicação. Passou a desenvolver-se bem, com bom ganho pôndero-estatural, adequado desempenho psíquico e social, porém com atraso global em todas as etapas do desenvolvimento motor, vindo a andar com a ajuda de muletas canadenses apenas aos 6 anos. A fala desenvolveu-se normalmente 10. O exame neurológico 11 revelou déficit motor, com tono aumentado nos membros inferiores, liberação piramidal global e estrabismo convergente bilateral. Trata-se, portanto, de criança com PC espástica, forma diplégica. Exame oftalmológico criterioso, realizado por especialista, revelou-se normal. A avaliação psicológica evidenciou desempenho compatível à idade cronológica.

O exame de funções corticais 9,15 não mostrou alterações no exame de linguagem, além das limitações impostas pelo fato de se encontrar a criança em processo de alfabetização. O ditado e a cópia evidenciaram tendência a escrever as palavras solicitadas com inclinação oblíqua em papel liso, sem pauta. As letras sofreram grande deformação e se tornaram quase ilegíveis quando as mesmas palavras foram escritas em papel pautado. O exame uas praxias ideativas e ideomotoras não revelou alterações. Entretanto, detectou-se apraxia construtiva, pois a criança não foi capaz de copiar losango e em alguns quadrados executou o traço vertical de maneira oblíqua. O exame das gnosias evidenciou domínio da lateralidade, das cores e boa discriminação auditiva e visual. Não havia déficit de memória. Os dados positivos encontrados na avaliação neuropsicológica levantaram a hipótese de haver distúrbio da função vísuo-espacial. Para melhor entendimento desta função resolvemos ampliá-la com o novo teste proposto.

A realização de exames complementares acrescentou dados para a correlação anatômico do distúrbio. Assim, o eletrencefalograma mostrou ritmos de fundo normais e espículas focais na região parietal esquerda; a tomografia computadorizada craniana evidenciou aumento dos cornos posteriores dos ventrículos laterais; a ressonância magnética nuclear de cabeça revelou T1 e T2 longos na substância branca ao longo dos ângulos dos ventrículos laterais, provavelmente representando gliose. O corpo caloso apresentou-se anormalmente delgado com exceção do joelho que era aproximadamente normal. A substância branca sei apresentou contraída, principalmente nas porções posteriores dos hemisférios, onde o cortéx chega ao nível dos ventrículos laterais que se apresentaram simétricos, porém de morfologia pouco habitual. A impressão do exame, sugerida pelos especialistas que o analisaram, é de que tais lesões representam gliose secundária a leucoencefalomalácia perinatal. Por esse exame, observamos haver componente de malformação do sistema nervoso central (SNC) representado pela hipogenesia de corpo caloso e componente, de etiologia vascular que se manilestou no período perinatal, representado pela gliose secundária a leucoencefalomalácia periventricular.

\section{RESULTADOS}

Os resultados podem ser observados nas figuras 1B, 1C, ID e 1E.

Nos testes individuais realizados no papel, preservou-se a distribuição) do traço no local solicitado; entretanto, o tamanho da cópia foi menor que o modelo e as linhas onduladas (Fig. 1B).

$\mathrm{Na}$ execução dos quatro traços em conjunto no papel, observamos que houve amontoamento num canto da página, a cópia foi menor que o modelo e as linhas foram ligeiramente onduladas com reversão em espelho do traço oblíquo para a esquerda (Fig. 1C).

$\mathrm{Na}$ realização dos traços no computador as dificuldades ficaram mais evidentes e surgiram outras, por exemplo, reversão em espelho de traços individuais e a não distribuição do traço no local solicitado (Fig. 1D).

$\mathrm{Na}$ execução do conjunto dos quatro traços, as dificuldades se acentuaram de tal forma que a criança não manteve as relações topográficas dos traços, fazendo-os ao acaso. Também houve amontoamento dos traços num oanto da tela (Fig. 1E). 
Comentaremos dois tópicos principais: o primeiro refere-se à existência de distúrbio da função vísuo-espacial em crianças com PC. O segundo propõe que o teste mostrou-se adequado para avaliar a função espacial e que o computador auxiliou na avaliação.

Buscamos nas teorias de Luria !3 e Piaget $^{16}$ os argumentos para embasar o primeiro tópico. Segundo Luria, as zonas terciárias da segunda unidade funcional do cérebro, correspondentes às áreas que se situam na fronteira entre os córtices occipital, temporal e parietal, têm como principal função a noção espacial. Essas áreas amadurecem depois de todas as outras zonas das regiões posteriores do córtex e se tornam plenamente operantes no sétimo ano de vida. Um distúrbio das zonas inferiores dos tipos correspondentes do córtex nos primórdios da vida deve conduzir inevitavelmente a desenvolvimento incompleto das zonas corticais superiores, ou áreas terciárias. Portanto, crianças com PC, cujo SNC sofre agressão precocemente no seu desenvolvimento, podem ter comprometida a formação das zonas corticais terciárias. Além disso, segundo a teoria de Piaget, as noções espaciais se desenvolvem pela interação e exploração do mundo feitas pela criança. A limitação motora que ocorre em muitas crianças com PC limita a experiência sensório-motora precoce e isto é considerado crítico para o desenvolvimento adequado da função espaciais.

A criança-propósito deste estudo, por sua história de vida e exames complementares, ilustra as duas proposições teóricas comentadas. Pela ressonância magnética nuclear constatou-se haver substrato anatômico justificando a idéia de dano cerebral precoce tanto pré-natal (malformação do corpo caloso) quanto perinatal (leucoencefalomalácia perí-ventricular). Este exame evidenciou comprometimento do SNC principalmente nas regiões posteriores dos hemisférios cerebrais, onde se localizam as zonas terciárias da segunda unidade funcional de Luria. Pela história de vida da criança, observamos haver também substrato fisiológico que justifica o precário desenvolvimento das noções espaciais em função da limitação motora.

O teste provou ser útil para a avaliação da função vísuo-espacial e o computador ampliou e aprofundou a investigação. Por esse teste extremamente simples, pudemos observar no presente caso todas as características dos desenhos de pacientes com agnosia espacial descritas por CritchleyG: linhas ondulosas, reversão em espelho, a cópia não preservou o tamanho do modelo e houve amontoamento dos desenhos num canto da página.

A realização dos traços em tela de computador permitiu o desdobramento em etapas, favorecendo o estudo destalhado de cada passagem e evidenciando os pontos de maior dificuldade. Com a máquina pode-se realizar avaliação «passo a passo», o que não é possível com lápis e papel. Além disso, a análise dos testes em computador foi mais detalhada, pois colhemos novas informações: constatamos que no papel houve dificuldade basicamente com o tamanho dos traços e seus ângulos, enquanto no computador, além dessas dificuldades, apareceram também dificuldades com a distribuição espacial e com noções de distância.

Concluindo, o teste aqui exposto é uma nova proposta para se avaliar a função vísuo-espacial e sua simplicidade permite o uso em computador, o que acrescenta elementos ao melhor entendimento do distúrbio.

\section{REFERÊNCIAS}

1. Abercombrie MJ. Perceptual and Visuo-Motor Disorders in Cerebral Palsy: a Survey of the Literature. Little Club Clinics in Developmental Medicine II. London: Medical Education and Information Unit of the Spastics Society in association with William Heineman Medical Books, 1964.

2. Ajuriaguerra J, Hécaen H. Le Cortex Cérébral. Paris: Masson, 1960.

3. Benton AL. Disorders of spatial orientation. In Vinken PJ, Bruyn GW (eds): Handbook of Clinical Neurology. Amsterdam: North-Holland, 1969, Vol 3, p 212.

4. Benton AL. Constructional apraxia an the minor hemisphere Confin Neurol 1967, $29: 1$.

5. Blair E, Stanley FJ. An epidemiological study of cerebral palsy in Western Australia, 1956-1975: III. Postnatal aetiology. Dev Med Child Neurol 1982, 24:575.

6. Critchley M. Disorders of spatial thought. In Critchley M: The Parietal Lobes. London: Hagner, Collier MacMillan 1953, p 326. 
7. De Renzi E. Disorders of spatial orientation. In Frederiks JAM (ed): Handbook of Clinical Neurology. Amsterdam: Elsevier 1985, Vol 1 p 405.

8. Eagle RS. Deprivation of early sensorimotor experience and cognition in the severely involved cerebral-palsied child. J Autism Dev Disorders 1985, 15 : 269.

9. Hartlage LC, Reynolds CR. Neuropsychological assessment and the individualization of instruction. In Hynd GW, Obrzut JE (eds): Neuropsychological Assessment and the School-Age Child. New York: Grune \& Stration, 1981, p 355.

10. Lefèvre AB. Exame Neurológico Evolutivo. São Paulo: Sarvier, 1972.

11. Lefèvre $A B$. $O$ exame físico e neurológico da criança. In Diament A, Cypel $S$ (eds) : Neurologia Infantil Lefèvre. Rio de Janeiro: Atheneu, 1989

12. Lord J. Cerebral palsy: a clinical approach. Arch Phys Med Rehab 1984, 65 : 542.

13. Luria AR. Higher Cortical Functions in Man. New York: Basic Books, 1966.

14. Nelson KB, Ellenberg JH. Antecedents of cerebral palsy: I. Univariate analysis of risks. Am J Dis Child 1985, $139: 1031$.

15. Obrzut JE. Neuropsychological procedures with school-age children. In Hynd GW, Obrzut JE (eds): Neuropsychological Assessment and the School-Age Child. New York: Grune \& Stratton, 1981, p 237.

16. Piaget J, Inhelder B. The Child's Conception of Space. New York: WN Norton, 1967.

17. Valente JA, Valente AB. Logo: Conceito, Aplicações e Projetos. São Paulo: McGraw-ᄀ -Hill, 1988 